Helgoländer wiss. Meeresunters. 17, 6-24 (1968)

\title{
Versuch einer Klassifikation industrieller Abfallprodukte in bezug auf die Möglichkeit einer Versenkung auf See
}

\author{
Gerhard Tomczak \\ Deutsches Hydrographisches Institut, Hamburg
}

\begin{abstract}
Attempt of a classification of industrial wastes with regard to a possible dumping at sea. Pollution of the sea by domestic and industrial wastes has become a danger to nearcoastal waters, and is extending now also into the open sea. These facts are the result of a world-wide inquiry circulated by UN specialized agencies; $71 \%$ of the coastal states replying to the inquiry stated that they had to deal with marine pollution affecting the marine fauna and flora, human health, and seaside resorts; $40 \%$ of the states proposed intergovernmental cooperation (an international convention) as a means of regulating and controlling the disposal of wastes. The present paper considers whether a classification of industrial wastes can be set up and serve as basis for negotiations. This classification should permit a clear decision whether or not a given substance may be disposed of in the sea. A classification based on the kind of the harmful effects and on certain groups of substances, which is presently being discussed on an international level, is considered insufficient for practical purposes. The same applies to a classification based on substance groups and sea areas, without distinguishing satisfactorily between the latter, and failing to allow for specific hydrographic conditions. The importance of the hydrography of different sea areas is exemplified, and a classification set up which renounces the use of substance groups but introduces a variety of criteria for assessing and arranging the individual substances in a more differentiated scheme of sea areas.
\end{abstract}

\section{EINLEITUNG}

Die menschliche Gesellschaft hat in den vergangenen zwei Jahrzehnten in zunehmendem Maße am Meer und an der Erforschung der vielen Probleme, die uns das Meer zu lösen aufgibt, Anteil genommen. Zahlreiche Veröffentlichungen in Zeitungen und populärwissenschaftlichen Zeitschriften, aber auch eine Reihe von Denkschriften in- und ausländischer Akademien und Ausschüsse sind ein Ausdruck hierfür. Uns Wissenschaftler, die wir in der Meeresforschung unsere Lebensaufgabe sehen, erfüllt dies verständlicherweise mit Freude und innerer Genugtuung. Nicht zuletzt erhoffen wir uns dadurch auch, daß durch die öffentliche Erkenntnis der Bedeutung des Meeres als einer Energie-, Mineral- und Rohstoffquelle die Bereitschaft der Gesellschaft dafür wächst, die mit der Meeresforschung befaßten Institute personell und finanziell in einer der Größe der Aufgaben entsprechenden Weise auszustatten. Das Wort vom „Zeitalter des Meeres", das in den Vereinigten Staaten in den politischen Diskussionen über die Förderung der Meeresforschung bewußt als gleichwertig neben das Wort vom 
"Atomzeitalter" gestellt wurde, beleuchtet diese von mir kurz umrissene Gegenwartssituation der Meeresforschung am besten. Nun gibt es im menschlichen Leben allerdings wohl kaum eine auf die Dauer ungetrübte Freude; so auch hier. Wir Meereskundler, die wir gerade mit Genugtuung das Interesse der öffentlichen Meinung an unserem Forschungsobjekt, dem Meer, wachsen sahen, mußten erkennen, daß dieses Interesse auch eine für uns negative Seite besaß.

Infolge der rasch wachsenden Produktion der großen Industriestaaten war die Industrie in diesen Ländern in eine schwierige Situation geraten. Es hatte sich gezeigt, daß das Aufnahmevermögen der Flüsse für die industriellen Abwässer und Abfallprodukte begrenzt war. Das biologische Selbstreinigungsvermögen der natürlichen Gewässer war - durch bloßen Augenschein zu erkennen - vielfach überzogen worden, so daß kostspielige Reinigungs- und Aufbereitungsanlagen geschaffen werden oder aber andere Wege zur Abfallbeseitigung gefunden werden mußten. Die Notwendigkeit hierzu und die Anteilnahme der Offentlichkeit am Meere fielen zeitlich nahe zusammen. Was lag näher als der Gedanke, die riesigen Wassermassen der Ozeane, die ein praktisch unendliches Selbstreinigungsvermögen durch biologischen Abbau, aber auch durch Austausch- und Vermischungsvorgänge zu versprechen schienen, für die Abfallbeseitigung auszunutzen. Große Industriewerke, die ihre Abwässer nicht mehr in Flüsse einleiten dürfen, verladen sie nun in Spezialschiffe und pumpen sie auf See über Bord; Großstädte verlegen Rohrleitungen von 1 bis $2 \mathrm{~m}$ Durchmesser in das Meer und leiten in einigen Kilometern vom Strand entfernt ihre Kloakenabwässer in das Meer. An diesen willkürlich herausgegriffenen Beispielen mag man erkennen, warum in zunehmendem Maße von den Fachwissenschaftlern der Meeresforschung den Chemikern, Biologen, Physikern und Geologen - aber auch von den Medizinern der Weltgesundheitsbehörde von der „Verschmutzung des Meeres" gesprochen wird. Inwieweit hierdurch tatsächlich ein ernstes Problem für diejenigen Nationen bereits entstanden ist, die an die Ozeane grenzen, lassen deren Antworten auf einen Fragebogen erkennen, der im Auftrage des Sekretariats einiger UN-Organisationen im vergangenen Jahr versandt worden war. Darin haben von 66 Nationen, die den Fragebogen beantworteten, 47 - das sind $71 \%$ - das Problem der marinen Verschmutzung in ihrem Hoheitsgebiet und darüber hinaus in der offenen See als schwerwiegend bezeichnet. In 36 Fällen wird eine akute Gefahr vor allem für die marinen Lebewesen und in 20 Fällen für die menschliche Gesundheit angegeben. $12 \mathrm{mal}$ wird aufgeführt, daß die Tätigkeit auf See - insbesondere die Fischerei - dadurch eingeschränkt würde, und in 19 Fällen ist davon die Rede, daß durch die Verschmutzung infolge übelriechender und häßlich gefärbter'Abwässer die Annehmlichkeiten der Erholung an'den Badestränden gemindert wird. Auf die Frage, welche Maßnahmen zur Verhütung einer weiteren Verschmutzung des Meeres ergriffen werden sollten, schlagen allein 25 Länder, also rund $40 \%$ der interessierten Staaten, eine zwischenstaatliche Kooperation oder eine internationale Konvention vor, etwa derjenigen zur Verhütung der Verschmutzung der See durch OOl entsprechend. In die gleiche Richtung zielt neben anderen Vorschlägen auch eine Empfehlung der von 13 Staaten mit Delegierten beschickten Arbeitsgruppe der Intergovernmental Oceanographic Commission, der IOC, die sich vor 5 Wochen in Paris zusammenfand, um über die Verschmutzung des Meeres zu beraten. Diese Gruppe, die für die 5. Vollversammlung der IOC im Oktober in Paris Empfehlungen 
auszuarbeiten hatte, schlägt unter anderem eine Zusammenarbeit der an diesem Problem interessierten UN-Organisationen vor, also der Food and Agricultural Organization (FAO), der Intergovernmental Maritime Consultative Organization (IMCO), der World Health Organization (WHO), der International Atomic Energy Agency (IAEA) und natürlich auch der IOC selbst. Ziel solcher Zusammenarbeit soll es unter anderem sein, die Ausbringung von Verschmutzungsstoffen nach einem festgelegten Plan - Art und Zusammensetzung der Stoffe, Menge, Verpackungsart usw. - zu melden und $\mathrm{zu}$ kontrollieren sowie Regeln dafür aufzustellen, was erlaubt sein soll und was nicht.

Ich habe mich etwas länger bei der Schilderung der allgemeinen Situation aufgehalten, um verständlich zu machen, warum ich überhaupt einen Versuch zur Klassifikation von Abfallstoffen erörtern möchte. Einerseits nämlich scheint mir nach Lage der Dinge, daß es uns Ozeanographen verwehrt ist, wie ein Don Quichote gegen jede Art der Abfallverbringung ins Meer in das Feld zu ziehen. Wir müssen erkennen, daß für manche Stoffe das Meer in begrenztem Umfang durchaus aufnahmefähig sein kann und die Industrie letztlich vor der harten Notwendigkeit der Abfallbeseitigung steht. Andererseits aber muß sich die Industrie im klaren darüber sein, daß eine unkontrollierte und unbeschränkte Verbringung aller ihrer Abfallprodukte ins Meer nicht möglich sein wird, ohne den Lebensraum der marinen Fauna und Flora in nicht wiedergutzumachender Weise zu stören und damit die Lebensinteressen der Menschheit zu schädigen. Es wird also nötig sein, nach vernünftigen Kompromissen zu suchen und deshalb zu versuchen, die Vielfalt der Abfallstoffe im Hinblick auf die Möglichkeit einer Versenkung im Meer in bestimmte Kategorien einzuteilen.

\section{KLASSIFIKATION NACH SCHADENSWIRKUNG}

Der erste - und soweit mir bekannt - bisher einzige international diskutierte Vorschlag zu einer Klassifikation der Abfallstoffe ordnet diese nach dem Grad der zu erwartenden Schadenswirkungen. Die Einteilung wurde von einer kleinen Arbeitsgruppe von Wissenschaftlern entworfen, die durch die wissenschaftlichen Beratungsgremien der IOC und der FAO mit einer ersten Bestandsaufnahme der Probleme der Meeresverschmutzung betraut worden waren. Sie wurde entsprechend einer Auswertung der Antworten der 66 Staaten auf den bereits erwähnten Fragebogen vom Sekretariat der IOC etwas geändert und entspricht in der in Tabelle 1 wiedergegebenen Form somit wohl am besten der augenblicklichen internationalen Situation.

Den verschiedenen Schadenswirkungen - angegeben in den senkrechten Spalten sind in den waagerechten Reihen verschiedene Gruppen von Abfallstoffen gegenübergestellt. Es werden fünf Kategorien der Schadenswirkung unterschieden: Gefahr für den Fortbestand oder das Wohlbefinden einmal der marinen Lebewesen, zum anderen des Menschen selbst, eine Behinderung der menschlichen Tätigkeit auf See - und zwar der Fischerei und der sonstigen Tätigkeit wie Schiffahrt, Ol- und Erdgasgewinnung und anderes - und eine Verminderung der Annehmlichkeiten, beispielsweise eine Verunreinigung oder Geruchsbelästigung an Badestränden usw. In den verschiedenen Reihen, die die Abfallstoffe zusammenfassen, sind jeweils Kreuze angegeben, wo auf Grund der Antworten zum Fragebogen am häufigsten Schadenswirkungen festzustellen 
Klassifikation industrieller Abfallprodukte

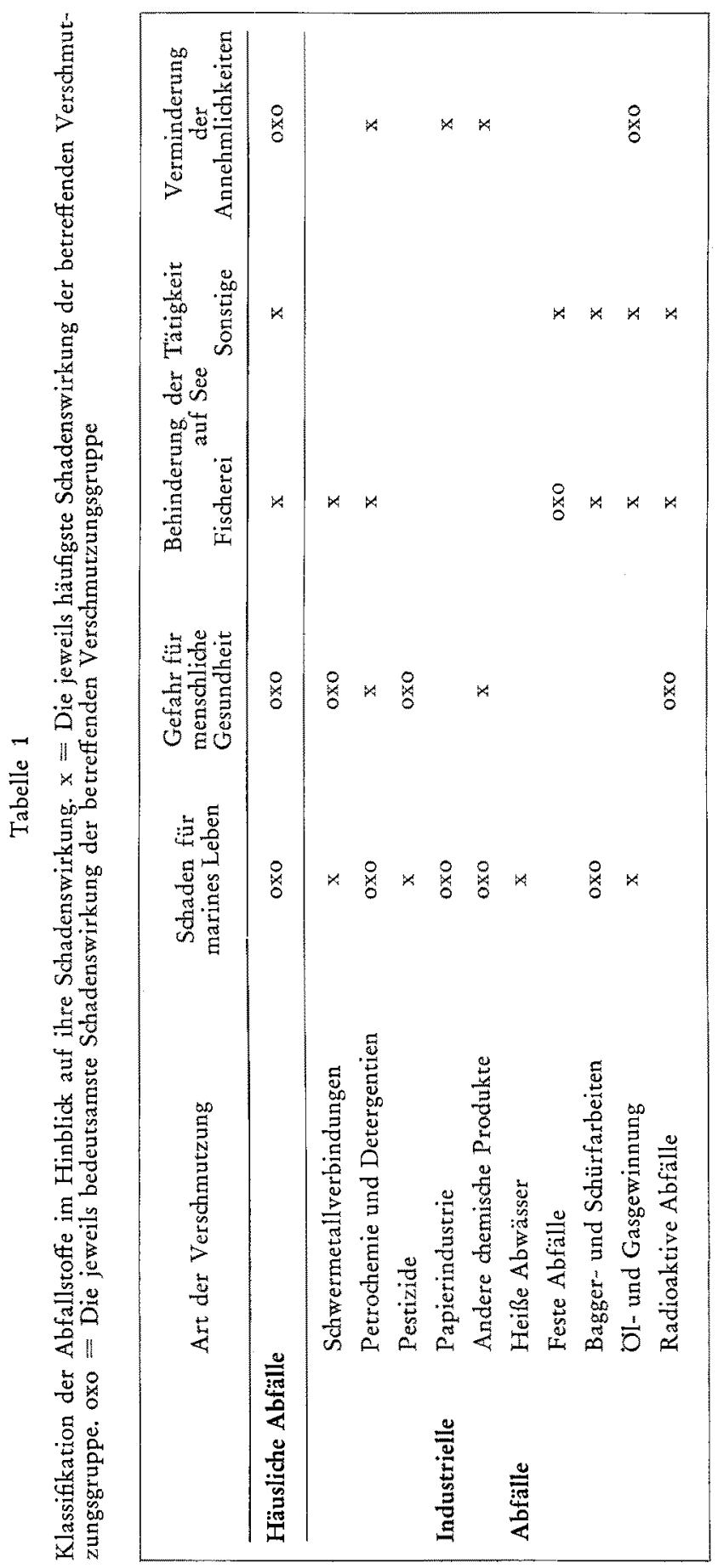


sind, und mit zwei Kreisen eingefaßte Kreuze, wo sie vermutlich den größten Schaden anrichten.

Das Schema soll hier nicht in allen Einzelheiten diskutiert werden. Ich will nur zu wenigen Gruppen einige Bemerkungen machen, und zwar vor allem im Hinblick darauf, wie diese nach wissenschaftlichen Gesichtspunkten zusammengestellte Klassifikation für die Praxis der Abfallbeseitigung verwendbar sein würde.

$\mathrm{Da}$ ist zunächst die Gruppe der Schwermetallverbindungen. Hier liegt eine besondere Gefahr (vgl. Tabelle 1, Spalte 2) darin, daß bestimmte, der menschlichen Ernährung dienende Arten, besonders die Filtrierer, durch Anreicherung von Schwermetallen wie Kupfer, Zink oder Quecksilber, die normalerweise im Meerwasser sonst nur in Spuren, in entsprechenden Abfallstoffen aber in größeren Mengen auftreten, eine unmittelbar giftige Wirkung auf den Menschen ausüben können. Außerdem kann hierdurch auch ein Massensterben der empfindlichen Fischlarven hervorgerufen werden.

Die Gruppe Petrochemie und Detergentien umfaßt alle Schäden durch O1 und ölhaltige Abwässer sowie die zur Beseitigung solcher Verschmutzungen häufig eingesetzten emulgierenden Detergentien. Die hauptsächliche Gefahr liegt dabei auf dem Gebiet des marinen Lebens infolge der toxischen Eigenschaften fast aller sogenannter Olbekämpfungsmittel; jedoch ist durch die Geschmacksbeeinflussung der Fische und durch die Möglichkeit der Anreicherung von karzinogenen Substanzen in bestimmten, dem menschlichen Verbrauch zugeführten Organismen auch eine Gefahr für die menschliche Gesundheit gegeben. In größeren Mengen auftretendes Öl schädigt außerdem das Fischereigeschirr und verschmutzt die Strände.

Die Schadenswirkung der Pestizide und Insektizide beruht wesentlich auf ihrem Gehalt an organischen Phosphorverbindungen und Chlorkohlenwasserstoffen; die der Abfälle aus der Papierindustrie auf dem hohen Sauerstoffverbrauch der sulfithaltigen Abflïsse. Feste Abfälle, hier besonders die Gruppe der Plastikgegenstände und -verpackungen, werden vor allem in den Küstengewässern in der Nähe der Hauptschiffahrtswege in zunehmendem Maße als schädlich und hinderlich für die Fischerei und Schiffahrt genannt.

Mit Ausnahme der Gruppe „andere chemische Produkte", die einen etwas unsicheren Sammelbegriff darstellt, sind - wie diese kurze Diskussion der Schadenswirkungen zeigen sollte - die Gruppen der Abfallstoffe gut definiert, und auch die Einteilung der Schadenswirkungen erscheint verständlich. Das Schema bildet sicher ein ausgezeichnetes Gerüst für ausführliche wissenschaftliche Diskussionen über die auf dem Gebiet der Einwirkung von Abfallstoffen noch nötigen Forschungsarbeiten. Es hilf aber nicht weiter, wenn es um die praktische Entscheidung geht, ob eine bestimmte Menge eines bestimmten Stoffes in einem bestimmten Seegebiet ausgebracht werden darf oder nicht.

Betrachtet man die Klassifikation unter solchen Gesichtspunkten, so sieht man, daß ihr eine Dimension fehlt, die den Ausbringungsort berücksichtigt. Bei Kenntnis des Ortes und der dort herrschenden hydrographischen Bedingungen könnte es gegebenenfalls möglich sein, einen bestimmten Stoff, der unter Laboratoriumsbedingungen eine schädliche Einwirkung auf marine Organismen aufweist, in begrenzten Mengen pro Zeiteinheit oder aber einmalig in die See zu verbringen, ohne damit größeren oder gar unwiderruflichen Schaden anzurichten. 


\section{KLASSIFIKATION NACH CHEMISCHEN STOFFGRUPPEN}

Um diese Lücke zu schließen, wurde von seiten der Industrie selbst eine Katalogisierung entworfen, die - das muß ich allerdings ausdrücklich betonen - nur als Diskussionsbasis im engeren Kreis gedacht war und daher keinerlei offiziellen Charakter hat. Es erscheint mir im Sinne einer systematischen Abgrenzung der Möglichkeiten aber wichtig, auch über diesen Vorschlag nachzudenken. Tabelle 2 zeigt das Schema, das ich entsprechend dem von der Industrie angegebenen Katalog entworfen habe. Es

Tabelle 2

Klassifikation der Abfallstoffe im Hinblick auf die möglichen Seegebiete

\begin{tabular}{|c|c|c|c|}
\hline $\begin{array}{l}\text { Gruppe der } \\
\text { Abfallstoffe }\end{array}$ & Küstenwasser & Schelfgebiet & Tiefe Meeresgebiete \\
\hline Feste Stoffe & $\begin{array}{l}\text { Lösliche oder feinteilige } \\
\text { unlösliche Stoffe mit } \\
\text { spez. Gewicht }>1,2 \\
\text { Ohne Wirkung auf } \\
\text { biologische Vorgänge }\end{array}$ & $\begin{array}{l}\text { Langsam lösliche oder } \\
\text { feinteilige unlösliche } \\
\text { Stoffe tnit spez. Gewicht } \\
>1,2 \\
\text { Ohne Wirkung auf } \\
\text { biologische Vorgänge } \\
\text { bei bestimmtem } \\
\text { Verdünnungsgrad }\end{array}$ & \\
\hline Beispiele: & $\begin{array}{l}\text { Anorganische } \\
\text { Mineralsalze } \\
\text { (Gips, NaCl, } \mathrm{BaSO}_{4} \text { ), } \\
\text { Kieselsäure }\end{array}$ & $\begin{array}{l}\text { Lösungsrïckstände bei } \\
\text { Herstellung anorgani- } \\
\text { scher Produkte, Metall- } \\
\text { hydroxyde [Al(OH) } \\
\text { Fe (OH })_{3} \text {, Calcium- } \\
\text { arsenat, Härtesalz, } \\
\text { Kohlehydrate, Schlamm } \\
\text { aus Kläranlagen] }\end{array}$ & \\
\hline $\begin{array}{l}\text { Flüssige oder } \\
\text { gelöste Stoffe }\end{array}$ & $\begin{array}{l}\text { Lösliche oder im Meer } \\
\text { dispergierbare Stoffe } \\
\text { Ohne Wirkung auf } \\
\text { biologische Vorgänge }\end{array}$ & $\begin{array}{l}\text { Lösliche oder im Meer } \\
\text { dispergierbare Stoffe } \\
\text { Ohne Wirkung auf } \\
\text { biologische Vorgänge } \\
\text { bei bestimmtem } \\
\text { Verdünnungsgrad }\end{array}$ & \\
\hline Beispiele: & $\begin{array}{l}\text { Anorganische } \\
\text { Mineralsalze } \\
{\left[\mathrm{Gips}_{2} \mathrm{NaCl}, \mathrm{Na}_{2} \mathrm{SO}_{4} \text {, }\right.} \\
\left.\mathrm{CaCl}_{2}\right]\end{array}$ & $\begin{array}{l}\text { Fäkalien, Abfallsäuren, } \\
\text { Phenole, Sdhwermetall- } \\
\text { salze [Cr, } \mathrm{Zn}, \mathrm{Mn}, \mathrm{Cu} \text {, } \\
\mathrm{Co} \text {, Alkalien, Farb- } \\
\text { stoffe, Pflanzenschutz- } \\
\text { mittel }\end{array}$ & \\
\hline $\begin{array}{l}\text { In Behältern } \\
\text { verpackte Stoffe }\end{array}$ & & & $\begin{array}{l}\text { Stoffe, die zu stark } \\
\text { verdünnt werden } \\
\text { müßten, um unschäd- } \\
\text { lich zu sein, oder die } \\
\text { heftig mit Meer- } \\
\text { wasser reagieren }\end{array}$ \\
\hline Beispiele: & & & $\begin{array}{l}\text { Chlorkohlenwasser- } \\
\text { stoffe, radioaktive } \\
\text { Substanzen }\end{array}$ \\
\hline
\end{tabular}


handelt sich offensichtlich um eine relativ einfache, auf die Praxis abgestimmte Einteilung. Den 55 Fächern des zuerst diskutierten Schemas, das nach wissenschaftlichen Gesichtspunkten geordnet war, stehen hier nur 5 Fächer gegenüber. Die Einteilung der Spalten gliedert den Ozean in drei Gebiete entsprechend den Meerestiefen: flache küstennahe Zonen, Schelfgebiete mit Wassertiefen bis zu $200 \mathrm{~m}$ und tiefe Ozeangebiete. Die Reihen unterscheiden nur zwischen festen, flüssigen und verpackten Abfallstoffen. Sicherlich wäre es wünschenswert - und es würde, wenn es möglich wäre, uns Meereskundlern die Arbeit wesentlich erleichtern - nach einem so einfachen Schema zu verfahren. Betrachtet man als Ozeanograph die von den Industriechemikern in den Feldern angegebenen Kriterien, nach denen die Abfallstoffe eingeordnet werden sollen, so muß man zu dem Schluß kommen, daß in bezug auf die hydrographischen Eigenschaften der verschiedenen Seegebiete zu wenig differenziert wird. Auch vom Standpunkt des Meeresbiologen dürften die zwei Unterscheidungen, die allein in diesem Schema vorgesehen sind, nicht genügen. Bei der Betrachtung des zuerst genannten Vorschlages zur Klassifikation auf Grund der möglichen Schadenswirkungen haben wir ja gesehen, wie vielfältig die biologischen Vorgänge abgestuft werden müssen. Daß die Kriterien vom Standpunkt der Meeresbiologen und -chemiker nicht ausreichen, machen auch die Beispiele deutlich, die in dem Katalog der Industrie vorgeschlagen wurden. Abfallstoffe wie Calciumarsenat, Härtesalz, Pflanzenschutzmittel, also Insektizide, und Kreosot haben eine ausgesprochen toxische Wirkung und sind - wenn überhaupt - ohne besondere Vorkehrungen zum Einbringen in Flachwassergebiete nicht geeignet.

Zweifellos gut an diesem Vorschlag ist der Gedanke, die Seegebiete und die Abfallstoffe als die beiden Koordinaten des Schemas anzunehmen und zusätzlich sowohl biologische als auch chemische Kriterien als weitere Variable einzuführen. Man muß aber bemängeln, daß die Stoffe zuwenig differenziert zusammengefaßt wurden und $\mathrm{da}$ auf die ozeanographischen Verschiedenheiten der einzelnen Seegebiete nicht eingegangen wird. Betrachten wir als Ozeanographen die Einteilung der Seegebiete in der yorgeschlagenen Kombination mit den Stoffgruppen, so müssen wir einschränkend auf die dreifache Ungleichung hinweisen: Küstenmeer $\neq$ Küstenmeer, Schelfgebiet $\neq$ Schelfgebiet und tiefes Meer $\neq$ tiefes Meer. Erst wenn man die Hydrographie der einzelnen Seegebiete berücksichtigt, kann man sie als eine Koordinate in ein Schema der Klassifikation übernehmen. Ehe ich auf eine solche beim Deutschen Hydrographischen Institut in der Verantwortung von Herrn Dr. WEICHART entwickelte Klassifikation eingehe, möchte ich auf einige Beispiele für die soeben genannten Ungleichungen eingehen.

\section{DIE HYDROGRAPHIE VERSCHIEDENER SEEGEBIETE}

In bezug auf die Küstenzonen kann ich mich kurzfassen. Hier genügt im Grunde ein Blick auf Atlanten und Seekarten, um die Verschiedenheiten der Küstengewässer sofort zu erkennen. Da gibt es bereits bei einem so begrenzten Nebenmeer wie der Nordsee langgestreckte, nahezu abflußlose Küstenabschnitte wie vor der belgischen oder vor der niederländischen Küste nördlich der Schelde- und Rheinmündung, es gibt 
Klassifikation industrieller Abbauprodukte
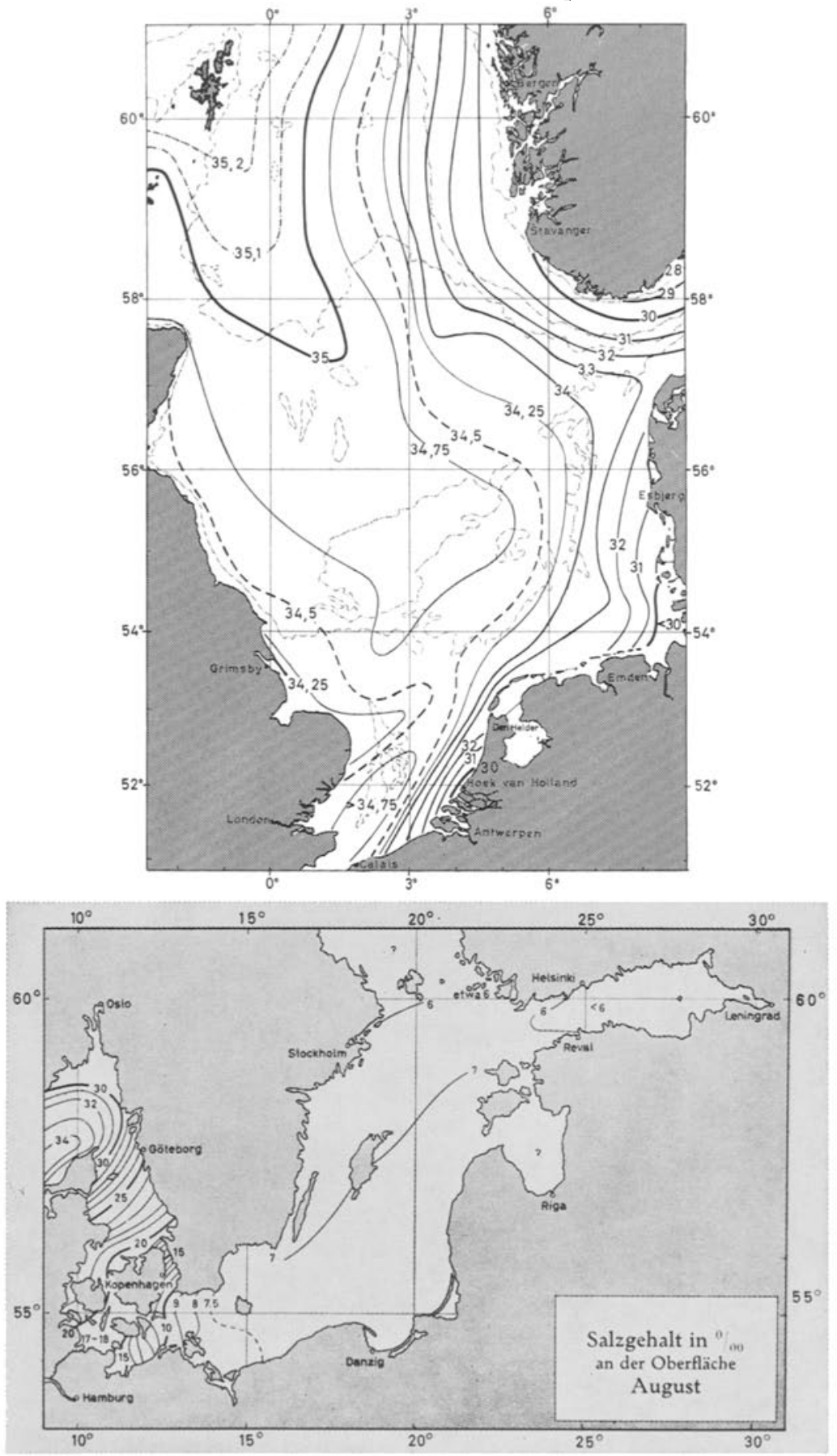

Abb. 1: Mittlerer Salzgehalt im August in der Oberflächenschicht von Nord- und Ostsee. (Aus: Deutsches Hydrographisches Institut, Seehandbücher Nr. 2006 und 2003) 
die davon völlig verschiedenen Wattengebiete hinter den westfriesischen Inseln und in der Deutschen Bucht, es gibt schließlich die Zone der Steilküsten und Fjorde in Norwegen und anders mehr. Daß es nicht gleichgültig sein kann, in welches dieser Küstengebiete man bestimmte Abfallstoffe einbringt, ist offenkundig.

Als Beispiel sei hier hingewiesen auf das Fischsterben, das vor einiger Zeit an der niederländischen Küste auftrat (KoRRINGA 1967). Ein Produzent hatte eine größere Menge Kupfersulfat, mit der er nichts mehr anzufangen wußte, am Strand bei Noordwijk in das Meer schütten lassen. Die entstehende giftige Lösung verdünnte sich trotz der Gezeiten und des Seegangs nur ganz langsam; sie vermischte sich keineswegs mit

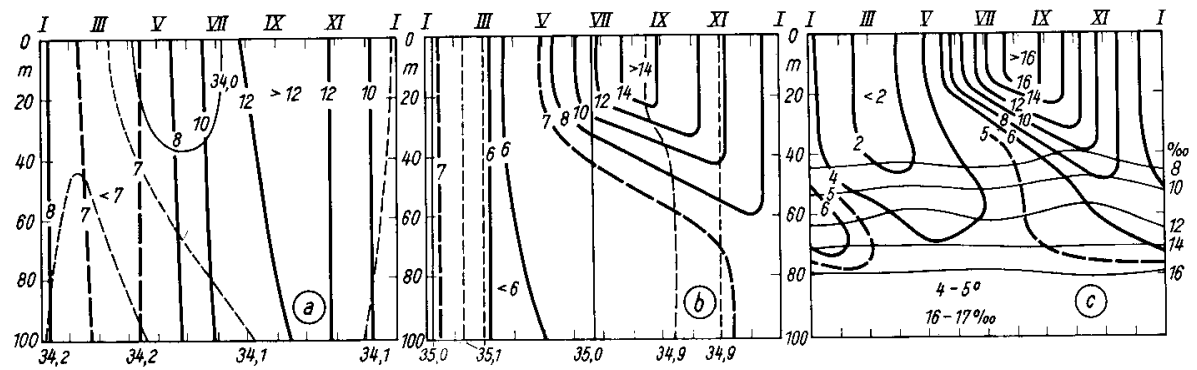

Abb. 2: Mittlerer Jahresgang von Temperatur und Salzgehalt von der Oberfläche bis zum Boden in Schelfmeeren. (a) Irische See, (b) Mittlere Nordsee, (c) Ostsee (Bornholm-Tief). (Aus: Dietrich \& Kalle 1957)

dem Meerwasser-Reservoir der Nordsee, sondern hielt sich in einem relativ schmalen Streifen an der Küste, wo sie mit der Strömung im Verlaufe von zwei Wochen langsam nordostwärts driftete. Erst ablandige Winde, die glücklicherweise gerade noch rechtzeitig einsetzten, um die Muschel-Kulturen im niederländischen Wattgebiet vor der Vernichtung zu bewahren, trieben die Lösung schließlich seewärts, wo sie sich allmählich zu einem unschädlichen Verdünnungsgrad vermischte. Sicherlich wäre der Vorgang anders verlaufen, wenn die Quelle der Verschmutzung im Abflußbereich eines großen Flusses gelegen hätte - was nun freilich, da es nur als Beispiel für die Behauptung Küstengewässer $\neq$ Küstengewässer dienen sollte, nicht so verstanden werden darf, als ob man Kupfersulfat durch Ausbringen in einem Flußmündungsgebiet vernichten könne!

Betrachtet man jetzt die zweite Behauptung: Schelfmeer $\neq$ Schelfmeer. Hier mögen uns ein paar Vergleiche zwischen der Nordsee und der Ostsee als Beweis dienen.

Abbildung 1 zeigt den mittleren Salzgehalt an der Oberfläche der Nord- und Ostsee. Bei letzterer wurde der Bottnische und Finnische Meerbusen allerdings nicht mitberücksichtigt. Beträchtliche Unterschiede sind $\mathrm{zu}$ erkennen. In der Nordsee ist der Salzgehalt relativ hoch; er variiert, abgesehen von den unmittelbaren Küstenzonen, aber nur gering zwischen etwa 30 und $35 \%$. Der größte Teil der Nordsee hat sogar weniger als $1 \%$ Unterschied im Salzgehalt aufzuweisen. In der Ostsee dagegen nimmt der Salzgehalt systematisch von $25 \%$ im Nordteil des Kattegat auf etwa $8 \%$ in der mittleren Ostsee bei Rügen ab. Die Abnahme findet dabei im wesentlichen im Kattegat statt, wo ein hoher horizontaler Salzgehaltsgradient beobachtet 


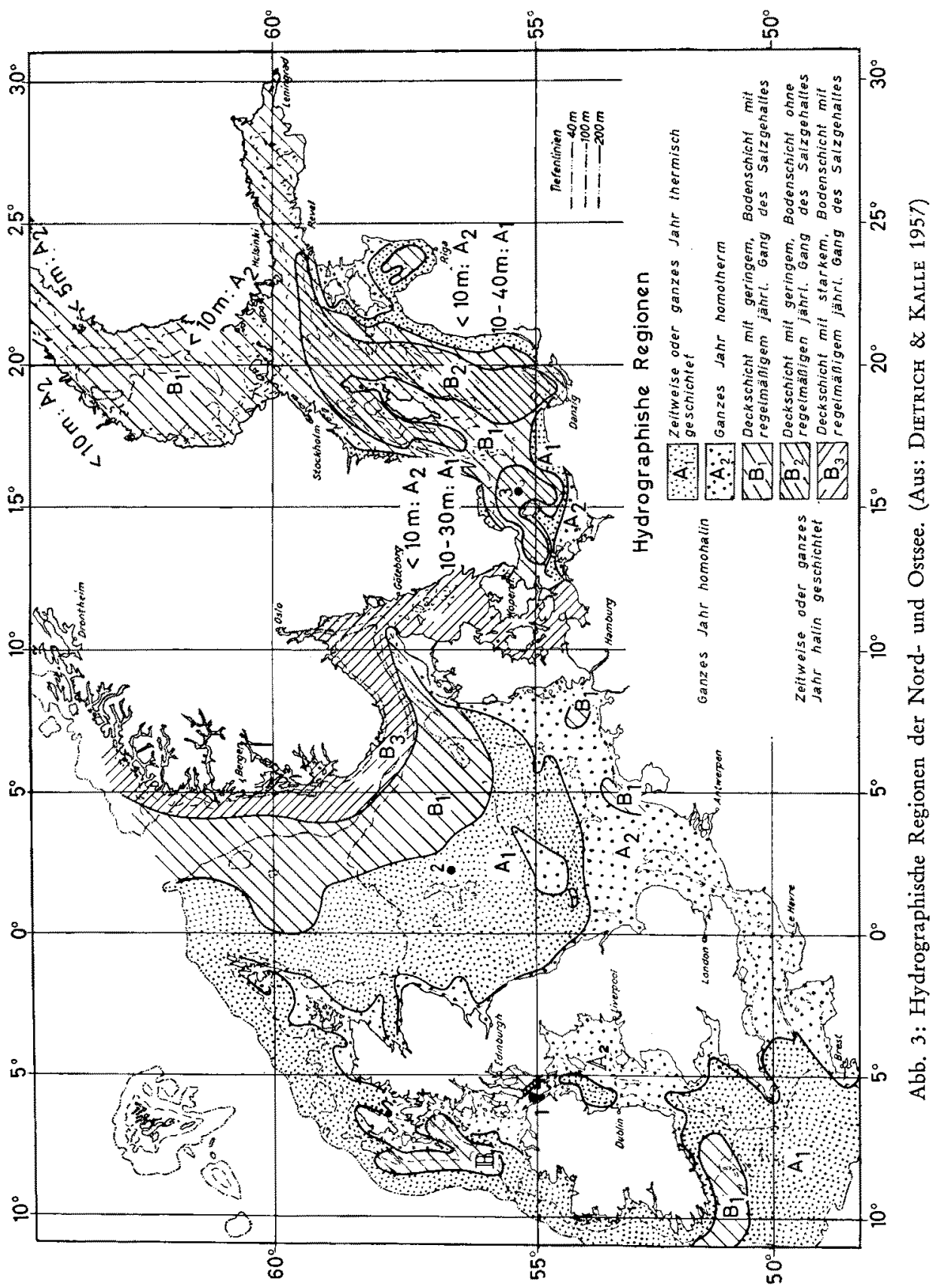


wird. Die eigentliche Ostsee weist dann zwar ähnlich wie große Teile der Nordsee kaum Unterschiede im Salzgehalt auf; jedoch ist der Betrag ( 8 bis $10 \%$ ) gegenüber der Nordsee mit den hohen Werten von 34 bis $35 \%$ sehr viel geringer. Weiter nach Osten hin nehmen die Werte übrigens noch weiter $a b$; im Nordteil des Bottnischen Meerbusens sind es nur noch etwa 2 bis $3 \%$.

In Abbildung 2 sind die mittlere Temperatur- und Salzgehaltsverteilung von der Oberfläche bis zum Boden und ihre Anderungen im Verlaufe des Jahres für drei Stationen dargestellt. Im linken Teil der Abbildung 2 befindet sich eine Station in der Irischen See. Infolge der hier sehr starken Gezeitenströme sind die Temperatur und der Salzgehalt nahezu das ganze Jahr über von der Oberfläche bis zum Boden gleich; das Gebiet ist völlig durchmischt. In der mittleren Nordsee, die durch die Station in der Bildmitte vertreten ist, sind die Gezeitenströme schwächer. Zwar erkennt man in bezug auf den Salzgehalt keinerlei Schichtung; die Temperatur ist während der Sommermonate jedoch in der oberen und unteren Wassermasse verschieden. Durch Einstrahlung erwärmtes Oberflächenwasser ist über kaltem Bodenwasser geschichtet, das seine Temperatur von 6 bis $7^{\circ} \mathrm{C}$ nahezu das ganze Jahr über behält. Die Ostsee schließlich ist durch die Station rechts im Bild vertreten. Zusätzlich zu einer thermischen Schichtung wie in der Nordsee finden wir in der gezeitenstromfreien Ostsee eine starke Salzgehaltsschichtung von etwa $40 \mathrm{~m}$ Tiefe. Die drei Isoplethendarstellungen machen deutlich, wie verschieden die drei Schelfmeere hydrographisch auf das Einbringen von Abfallstoffen reagieren müssen. Dabei ist zu bedenken, daß die thermische Schichtung keineswegs im gesamten Seegebiet gleichmäßig ausgebildet ist.

Berücksichtigt man den Salzgehalt an der Oberfläche und die thermischen und halinen Schichtungen, so verwundert es wohl nicht, wenn man zu dem Ergebnis kommen muß, daß nicht nur die Schelfmeere Nord- und Ostsee sich untereinander hydrographisch verschieden verhalten, sondern daß auch innerhalb dieser Seegebiete selbst verschiedene hydrographische Regionen auftreten. Dietrich hat deshalb die in $\mathrm{Ab}$ bildung 3 dargestellten hydrographischen Regionen abgegrenzt (vgl. DiETRICH \& KALLE 1957).

Die Abgrenzung ist nach dem thermischen Verhalten (Gruppen A1 und A2) und nach dem Jahresgang des Salzgehaltes (Gruppen B1 bis B3) vorgenommen worden. Ohne bei der Betrachtung dieser Abbildung auf weitere Einzelheiten einzugehen, wird doch sofort deutlich, daß die Nordsee im West- und Südteil wesentlich durch den jährlichen Temperaturgang, die Ostsee dagegen durch unterschiedliches Verhalten der halinen Veränderungen gekennzeichnet sind. Der Küstenstreifen vor der norwegischen Küste ist hydrographisch dem Kattegat und der westlichen Ostsee vergleichbar, während die östliche Nordsee außerhalb der unmittelbaren Küstenzone wiederum ein vergleichbares Gebiet in der Ostsee erst im Finnischen und Bottnischen Meerbusen findet.

Auf die Verschiedenheit der Strömung und deren Einflüsse auf die Vertikalverteilung der Temperatur und des Salzgehaltes hatte ich schon hingewiesen. Dabei war verschiedentlich von der durch die Gezeitenströme in der Nordsee bedingten Durchmischung die Rede gewesen. Auch tritt in den Wintermonaten durch die Abkühlung der Oberflächenwassermassen regelmäßig eine Vertikalkonvektion auf, die für einen durchgreifenden Vertikalaustausch des Nordseewassers sorgt. Abbildung 4 soll deutlich machen, daß auch in bezug auf die Vertikalkonvektion ein bedeutsamer Unter- 
schied zwischen den beiden Schelfmeeren existiert. Dargestellt ist die Vertikalverteilung von Temperatur, Salzgehalt, Sauerstoff und Phosphat an einer Station im Gotland-Tief zu zwei verschiedenen Zeiten, im Mai 1931 und Oktober 1935. Der normale jahreszeitliche Rhythmus im hydrographischen Aufbau der Wassermassen wird nach Kalle (1943), von dem diese Zustandskurven der Station im Gotland-Tief stammen, durch einen langfristigen Prozeß der Wasserumschichtung überlagert. Das 1931 in der

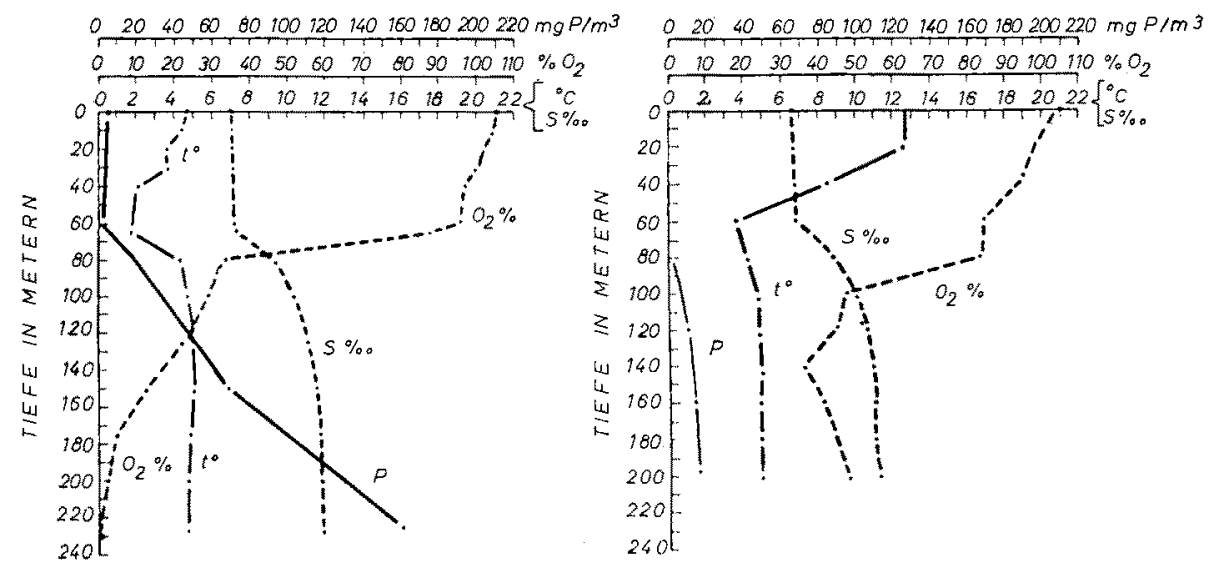

Abb. 4: Verteilung von Temperatur, Salzgehalt, Phosphat und Sauerstoff im Gotland-Tief (Mai 1931 und Okrober 1935). (Aus: Dietrich \& Kalle 1957, nach Kalle 1943)

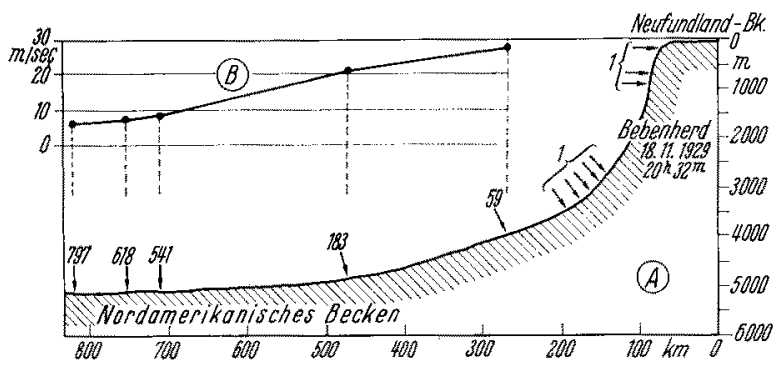

Abb. 5: Kabelbrüche südlich der Neufundlandbank bei dem Erdbeben am 18. November 1929. (Aus: Dietrich \& Kalle 1957)

Tiefe noch erkennbare sauerstoffarme, aber phosphatreiche Wasser hat im Verlaufe dieses Prozesses im Zeitraum 1933 bis 1934 durch einen Einbruch schwerer ozeanischer Wassermassen aus dem Skagerrak eine völlige Umschichtung erfahren und wurde durch sauerstoff reiches, dagegen phosphatarmes Frischwasser ersetzt. Die dabei aus den Tiefen an die Oberfläche kommenden Nährstoffe bedeuten für die Oberflächenschichten eine Düngung, die ihren Einfluß auf die biologischen Vorgänge entsprechend geltend macht. Dieser Prozeß ist in der Nordsee, in der eine regelmäßige winterliche Konvektion für die Umschichtung der Wassermassen sorgt, nicht denkbar. 
Damit verlasse ich die Überlegungen, die zeigen sollen, daß man bei einer Klassifikation nicht einfach von Schelfmeeren sprechen darf, ohne zugleich zu sagen, welches Seegebiet gemeint ist. Bei dem Nachweis schließlich der dritten Behauptung - tiefer Ozean + tiefer Ozean - möchte ich mich nunmehr nur kurz aufhalten. Immerhin sollen zwei Beispiele zeigen, mit welchen Ubberraschungen man im tiefen Ozean rechnen muß.
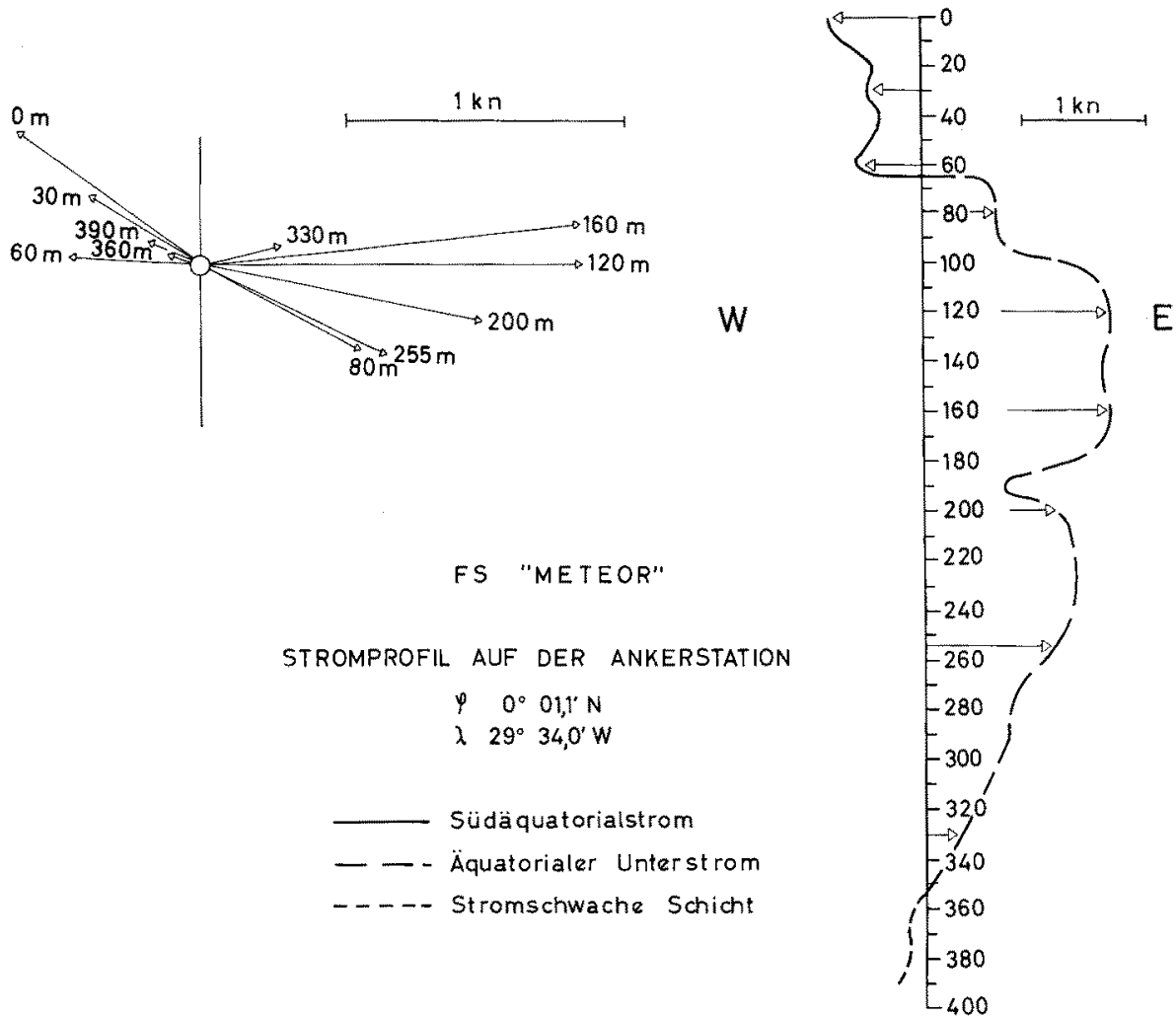

Abb. 6: Stromprofil auf einer vierwöchigen Ankerstation des FS „Meteor" am Aquator während der „Atlantischen Expedition Meteor, IQSY, 1965“. (Aus: Archiv des Deutschen Hydrographischen Instituts, Abteilung Meereskunde)

Abbildung 5 stellt ein Tiefenprofil des Seegebietes südlich der Neufundlandbank dar, das bis etwa $5000 \mathrm{~m}$ Tiefe reicht. Am 18. November 1929 ereignete sich am Kontinentalabfall etwa in $2000 \mathrm{~m}$ 'Tiefe ein Seebeben, das 13 Kabelbrüche von Tiefseekabeln zur Folge hatte. Acht von ihnen erfolgten nahezu gleichzeitig mit dem Beben, die anderen in zeitlichem Abstand, der mit zunehmender Entfernung vom Bebenherd wuchs. Die an dem Profil angegebenen Zeiten bedeuten die Minute des Kabelbruches nach dem Zeitpunkt des Bebens. Man weiß jetzt, daß die Ursache der verspätet eintretenden Brüche in Schlammströmen zu suchen ist, die durch Rutschungen am Kontinentalabfall in der Umgebung des Bebenherdes in Gang gesetzt worden waren und lawinenartig zunehmend beträchtliche Geschwindigkeiten erreicht hatten. In der oben links im Bild eingezeichneten Linie ist zu erkennen, wie die Geschwindig- 
keit von $28 \mathrm{~m} / \mathrm{sec}$ (d.h. $100 \mathrm{~km} /$ Stunde) in etwa $180 \mathrm{~km}$ horizontaler Entfernung vom Bebenherd allmählich auf $6 \mathrm{~m} / \mathrm{sec}$ in über $700 \mathrm{~km}$ Entfernung abgenommen hat. Ein so klarer Nachweis von solchen beträchtlichen Geschwindigkeiten entlang dem Meeresboden mit entsprechenden katastrophalen Folgen für Seekabel oder auch andere, beispielsweise dort abgelagerte Abfallbehälter, war nur durch die genaue zeitliche Festlegung der Kabelbrüche möglich. Das Beispiel zeigt, daß man - je nach der Morphologie - im tiefen Ozean mit katastrophalen Ereignissen und Angriffen auf abgelagerte Abfälle wird rechnen müssen.

Ein letztes Beispiel schließlich soll sich noch mit den Strömungen im Ozean befassen. Während man im allgemeinen damit rechnen kann, daß die winderzeugten Meeresströmungen bis zu einer Tiefe von einigen hundert Metern hinabreichen und darunter nur höchstens schwache Strömungen im Zuge eines Gesamtzirkulationssystems der Ozeane vorhanden sind, wurde vor etwa 15 Jahren entdeckt, daß unter der Meeresoberfläche - in Tiefen von wenigen Dekametern bis etwa $300 \mathrm{~m}$ - ganz beträchtliche Stromsysteme existieren können. Solche Ströme sind im Pazifischen, Indischen und Atlantischen Ozean genau unter dem Aquator gefunden worden, wo sie in einem etwa $250 \mathrm{~km}$ breiten Streifen von Westen nach Osten fließen. Aus den dabei auftretenden Geschwindigkeiten, die bis zu $2 \mathrm{~m} / \mathrm{sec}$ betragen können, und dem ermittelten Querschnitt ist leicht zu berechnen, daß die in diesen Strömen, die man äquatoriale Unterströme nennt, transportierten Wassermassen nicht viel hinter dem durch den Golfstrom bewirkten Wassertransport zurückstehen!

Abbildung 6 zeigt ein vertikales Stromprofil, das wir auf dem Forschungsschiff "Meteor" bei $30^{\circ} \mathrm{W}$ am Äquator gemessen haben, als das Schiff im Herbst 1965 dort vier Wochen verankert lag.

Eine oberflächennahe Schicht ist zu erkennen, in der die vom Passat nach Westen geführten Wassermassen Geschwindigkeiten von $50 \mathrm{~cm} / \mathrm{sec}$ aufweisen. In $66 \mathrm{~m}$ Tiefe erfolgt dann plötzlich eine Stromumkehr nach Osten; dieser Strom erreicht in $130 \mathrm{~m}$ Tiefe seine höchsten Geschwindigkeitswerte von $80 \mathrm{~cm} / \mathrm{sec}$ und geht schließlich in $355 \mathrm{~m}$ Tiefe wieder in eine langsame westliche Strömung über.

\section{KLASSIFIKATION NACH PHYSIKALISCHEN, CHEMISCHEN UND HYDROGRAPHISCHEN GESICHTSPUNKTEN}

Ich schließe hier meinen Exkurs in die Ozeanographie $a b$, der darauf abzielte, nachzuweisen, daß eine Katalogisierung von Abfallstoffen nicht ohne Kenntnis der jeweiligen hydrographischen Verhältnisse am vorgesehenen Ausbringungsort erfolgen kann. Damit ist aber eine Vielzahl von Parametern zu berücksichtigen, die es nach unserer Meinung nicht mehr zuläßt, die Abfallstoffe summarisch in Gruppen einzuordnen. Aus diesen Gründen haben wir beim Deutschen Hydrographischen Institut uns zu einem anderen System der Klassifikation von Abfallstoffen entschlossen. Dieses ergab sich aus der Praxis heraus, nachdem wir für eine Reihe von Anfragen zur Abfallbeseitigung im Meer zusammen mit dem Institut für Küsten- und Binnenfischerei in der Bundesforschungsanstalt für Fischerei, Hamburg, Gutachten oder auch nur Arbeitsunterlagen ausgearbeitet hatten. Es ist dies das System, über dessen Erweiterung 
WeICHART (1968) berichtet hat. Ich kann es mir deshalb ersparen, auf Einzelheiten einzugehen, sondern will an Hand der Tabelle 3 nur das Prinzip erläutern. In den Spalten treten wieder die Küstengewässer, die Schelfgebiete und tiefe Meeresgebiete auf. Diesmal jedoch in Unterscheidungen gemäß den Zusätzen: ohne Vorbehalt, leichte Vorbehalte, starke Vorbehalte, Tiefen $>1500 \mathrm{~m}$, Tiefen $>3000 \mathrm{~m}$. Durch diese Zusätze ist die Möglichkeit gegeben, hydrographische Gesichtspunkte bei der Wahl des Seegebietes zu berücksichtigen. In den Reihen treten weiterhin keine Stoffgruppen mehr auf. Das Schema nennt lediglich eine Reihe von Merkmalen zur Beurteilung und Einordnung der Einzelstoffe: Menge, physikalische und chemische Eigenschaften, biologische Wirkung und schädliche Einflüsse. Wir glauben, daß man nur so dem jeweiligen Fall gerecht werden kann, sowohl um den Befürchtungen der Ozeanographen im Hinblick auf die Verschmutzung des Meeres als auch den Wünschen und Notwendigkeiten der Industrie gerecht zu werden. In jeder Spalte ist zur Erläuterung lediglich ein Beispiel angegeben. Tatsächlich verfügen wir in der Praxis über wesentlich mehr Fälle, wobei wir bekennen müssen, daß wir leider offensichtlich längst nicht vollkommen informiert sind, welche Stoffe und welche Mengen bisher von der deutschen und natürlich noch weniger, welche Stoffe von der ausländischen Industrie in das Meer eingebracht werden.

Mit dieser Feststellung nähere ich mich dem Schluß meiner Ausführungen. Das internationale Recht gibt zur Zeit keine Handhaben, das Ausbringen von Abfallstoffen auf hoher See außerhalb der Hoheitsgewässer der einzelnen Staaten in irgendeiner Form zu beeinflussen; in den Hoheitsgewässern selbst wird dies durch die Rechtsprechung der einzelnen Staaten, die aber leider sehr unterschiedlich ist, geregelt. Es liegt im Interesse der menschlichen Gesellschaft, hier Abhilfe zu schaffen. Wir müssen verhindern, daß das Meer durch unbedachtes Einbringen von Abfallstoffen unwiderruflich verschmutzt wird, und wir müssen erreichen, daß für das Einbringen solcher Stoffe in allen Ländern die gleichen Voraussetzungen geschaffen werden, so daß die Industrien aller Länder unter einigermaßen gleichen Bedingungen arbeiten können. Es kann dabei geschehen, daß man um der Erhaltung eines gesunden Meeres willen strenge Maßstäbe anlegen muß, die der Industrie hohe Investitionen auferlegen. Wir als Ozeanographen sind aber der Meinung, daß solche Investitionen notfalls von der Allgemeinheit in Gestalt höherer Preise für die Produkte getragen werden müssen als ein Tribut, den die Menschen in den Industrieländern für den steigenden Lebensstandard zu entrichten haben. Wir sind jedenfalls dankbar, daß das Gespräch über die Verschmutzung des Meeres auf nationaler und internationaler Ebene begonnen hat, wie im Arbeitskreis „Abwassereinflüsse im Küstengebiet“ der Deutschen Forschungsgemeinschaft, hier auf diesem internationalen Symposium über „Biologische und hydrographische Probleme der Wasserverunreinigung in der Nordsee und den angrenzenden Gewässern", in der Arbeitsgruppe „Marine Verschmutzung" der IOC oder in der FAO. Wir erkennen mit Freude, daß die Bereitschaft zum internationalen Gespräch und zur Ausarbeitung regionaler, bilateraler oder internationaler Abkommen ständig im Wachsen ist und wollen nach besten Kräften daran mitarbeiten, gerade auch, um der Industrie Klarheit über ihre zukünftigen Möglichkeiten der Abfallverbringung zu schaffen. 

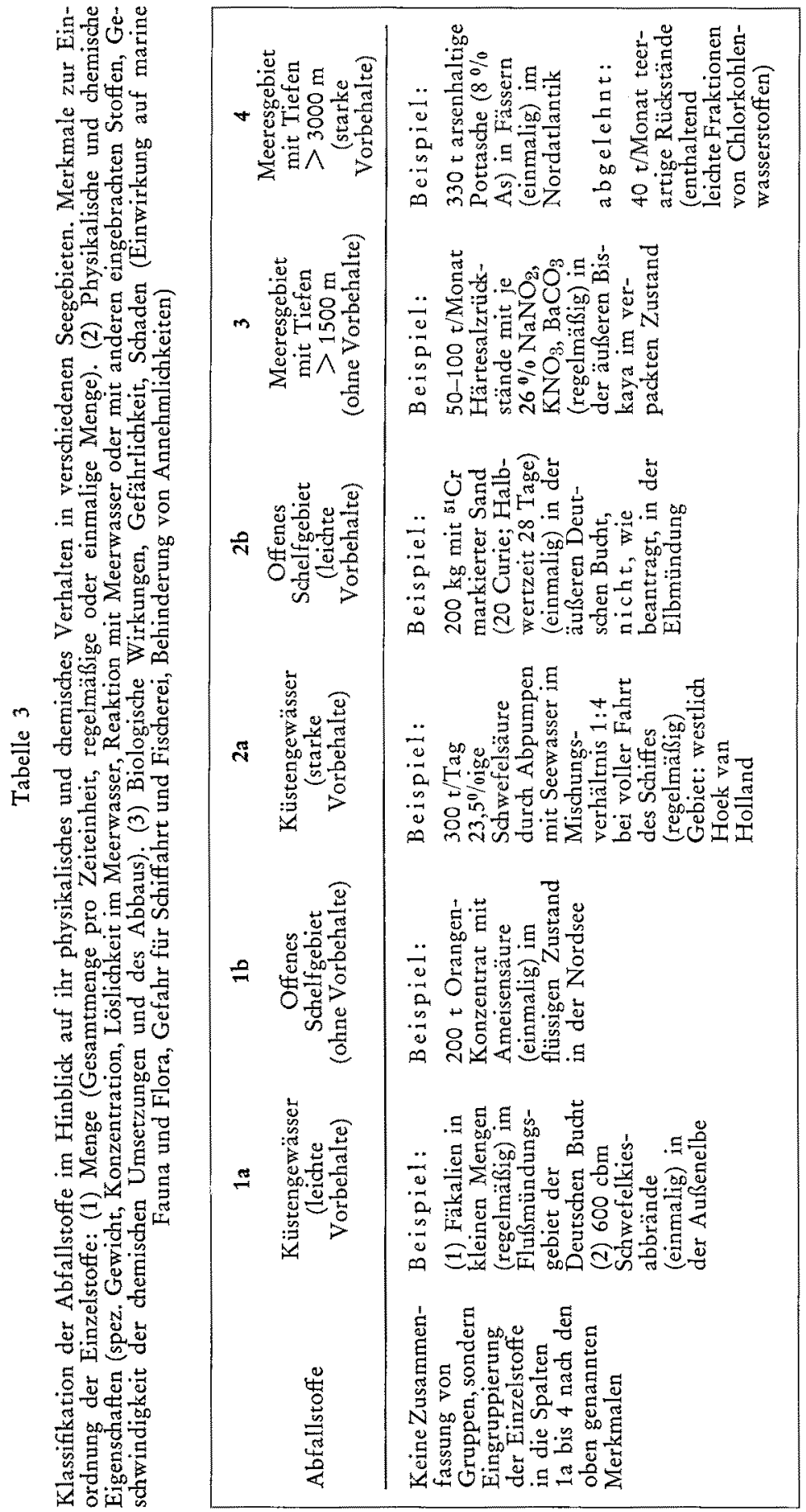


\section{ZUSAMMENFASSUNG}

1. Die Verschmutzung des Meeres ist ein internationales Problem. Beweis hierfür sind die Antworten der Küstenstaaten auf eine Umfrage von UN-Organisationen. 40\% der interessierten Staaten wünschen eine zwischenstaatliche Kooperation oder eine Konvention zur Lösung dieses Problems.

2. Eine Klassifikation, die von den wissenschaftlichen Beratungsgremien der IOC und FAO entworfen wurde, ist geeignet, die wissenschaftliche Diskussion über noch zu lösende Forschungsaufgaben zu fördern. Sie erscheint jedoch nicht geeignet für die praktische Regelung der Fragen, die mit der Versenkung von Abfallstoffen zusammenhängen.

3. Eine in Kreisen der chemischen Industrie als Arbeitsbasis entworfene Klassifikation berücksichtigt die Hydrographie des Meeres nicht genügend.

4. Die verschiedenen hydrographischen Bedingungen von Küstengewässern, Schelfgebieten und den Gebieten mit großen Meerestiefen werden an Beispielen gezeigt.

5. Es wird eine Klassifikation entworfen, die eine differenzierte Einteilung der Meeresgebiete vorsieht und auf die Zusammenfassung der Abfallstoffe in Stoffgruppen verzichtet. Statt dessen werden als Merkmale zur Beurteilung und Einordnung der Einzelstoffe eingeführt: Menge, physikalische und chemische Eigenschaften, biologische Wirkung und schädigende Einflüsse.

\section{ZITIERTE LITERATUR}

Dretrich, G. \& Kalle, K., 1957. Allgemeine Meereskunde. Borntraeger, Berlin, 492 pp.

KALLE, K., 1943. Die große Wasserumschichtung im Gotland-Tief vom Jahre 1933/34. Anmln Hydrogr., Berlin 71, 142-146.

Korringa, P., 1967. Mündliche Mitteilung.

Tomczak, G. \& Goedecke, E., 1964. Die thermische Schichtung der Nordsee auf Grund des mittleren Jahresganges der Temperatur in $1 / 2^{\circ}-$ und $1^{\circ}$-Feldern. Dt. bydrogr. Z. (Erg. H. R. B.) 8, 1-182.

WeICHART, G., 1968. Veränderungen des Meeres durch Abfälle und die darauf basierende Einführung von 5 Gefahrenklassen für das Einbringen von Abfällen ins Meer. Helgoländer wiss. Meeresunters. 17, 398-410.

\section{Diskussion im Anschluß an den Vortrag TomczaK}

WEBER: Für den Klassifizierungsvorschlag der Industrie ist die Berïcksichtigung der Konzentration des zu versenkenden Stoffes wesentlich. In normalen Konzentrationen giftig wirkende Stoffe können unterhalb einer bestimmten Grenze für Organismen harmlos sein. So spricht man für Schwermetallzonen in Flüssen von einer unteren Grenze der Schädlichkeit von etwa $1 \mathrm{mg} / \mathrm{l}$. Auch im Meer dürfte eine untere Grenze existieren, deren Ermittlung aber noch langwierige Forschungen erfordern wird. Beim Versenken von Stoffen kommt es daher darauf an, diese Grenze durch entsprechende Verdünnung zu unterschreiten. Wie man eine bestimmte Verdünnung technisch erreichen kann, wurde in Modellversuchen in Holland geklärt. Danach sind Verdünnungen 1:7000 bis 1:10000 im Schraubenstrom hinter einem Schiff erreichbar. Nach dieser Anfangsverdünnung erfolgt eine weitere langsame Endverdünnung durch Strömungen und Bewegungen des Meerwassers. Die Industrie hat ein großes Interesse daran, daß Forschun- 
gen zu meeresbiologischen Fragen in Gang kommen. Sie hat ihren Willen zur Zusammenarbeit bereits mehrmals zum Ausdruck gebracht (z. B. in Bremerhaven anläßlich des Kolloquiums über Abwassereinflüsse in Küstennähe). Hohe Kosten der Abwasserreinigung können ein Produkt sehr stark belasten. Unter Umständen muß dann seine Herstellung wegen zu hoher Kosten eingestellt werden. In einem anderen Land oder Kontinent ist seine Herstellung jedoch vielleicht lohnend, weil dort weniger scharfe Auflagen zur Abwasserreinigung gemacht werden.

Tomczak: (1) Der Verdünnungsgrad wird in unserem Schema für die praktische Beratung durchaus berücksichtigt. Er darf jedoch nicht absolut gesehen werden. Beispielsweise können bei Schwermetallen, die Herr WEBER erwähnte, bestimmte Organismen die Stoffe auch aus den sich zur Ungefährlichkeit verdünnten Substanzen so anreichern, daß sie plötzlich gefährlich in den Kreis der menschlichen Nährstoffe zurückkommen. (2) Die Kostenfrage: Ich erwähnte, daß die Verhandlungen hier so gefühtt werden müssen, daß gleiche Bedingungen für alle Länder gelten, so daß die Industrien der verschiedenen Länder untereinander konkurrenzfähig bleiben.

GERLACH: Sie schildern die empirische Methode, auf Grund bisheriger Abfallverbringungen in das Meer und den daraus resultierenden Erfahrungen zu einer Klassifikation industrieller $\mathrm{Ab}$ wässer zu kommen. Wie beurteilen Sie die Aussicht, in absehbarer Zeit einen Katalog aller wahrscheinlicher Abfallstoffe und der Möglichkeit ihrer Verbringung bzw, eines notwendigen Verbots zu erarbeiten?

ToмсZAK: Wir müssen von den Tatsachen ausgehen, daß die Industrie bestimmte Abfälle loswerden muß. Eine allgemeine Zusage, bestimmte Stoffe, die sich an sich als ungefährlich erwiesen haben, in das Meer zu verbringen, ist deshalb nicht gut, weil sie unter Umständen in Verbindung mit anderen, ebenfalls ausgebrachten Stoffen durch chemische Reaktion gefährliche Auswirkungen zeigen können. Es wird auf die Ausführungen von Dr. Weichart ( Helgoländer wiss. Meeresunters. 17, 398, 1968) verwiesen, der hierfür ein Beispiel bringen wird. Sicher ist, daß für die praktische und wissenschaftliche Anwendung des Schemas viele Grundsatzfragen zu klären sind, für die das zuerst genannte Schema, wie ich in meinem Vortrag ausführlich sagte, eine sehr gute Grundlage darstellt.

KrNNE: Klassifikationen haben Vor- und Nachteile - und ihre Gefahren. Die Vorteile liegen vielfach in der durch schematische Vereinfachungen verbesserten Obersichtlichkeit und leichteren Handhabung, die Nachteile in einer gewissen Starrheit und Unspezifität; die Gefahren liegen vor allem in einer unkritischen Anwendung. Ich möchte meinen, daß man auf der hier durch Herrn ToмсzaK als Diskussionsbasis präsentierten Klassifikation durchaus aufbauen kann. Eine größere Elastizität und Spezifftät könnte erreicht werden, wenn man die lokalen Gegebenheiten, die individuelle Situation, stärker berücksichtigen würde. Sicher wäre eine zu globale und zu stark vereinfachte Einteilung der ins Meer zu verbringenden Abfallsubstanzen gefährlich. Man sollte die Tatsache nicht aus den Augen verlieren, daß ins Meer geleitete Abwasserinhaltsstoffe im allgemeinen keineswegs wohldefinierte chemische Einzelsubstanzen darstellen, sondern meist nur unvollkommen analysierte Gemische. Die verschiedenen Gemische könnten sich in ihren physiologischen und ökologischen Wirkungen modifizieren: verstärken, neutralisieren oder abschwächen. Außer Untersuchungen über die biologischen Konsequenzen bestimmter Einzelsubstanzen unter definierten Versuchsbedingungen müssen wir daher Untersuchungen über die Wirkungen verschiedener Substanzkombinationen anstellen. Sicher sind auch die chemischen Interreaktionen zwischen verschiedenen Abwasserkomponenten von Wichtigkeit. Schließlich ist das Schicksal der ins Meer verbrachten Abwasserinhaltsstoffe zu berücksichtigen; sie behalten $\mathrm{ja}$ in der Regel ihre chemische Identität nicht bis in alle Ewigkeit bei, sondern werden durch eine Reihe von Prozessen - zum Beispiel durch Biodegradation - modifiziert; sie können ferner durch hydrographische Faktoren oder durch Bioakkumulation in bestimmten Meeresgebieten oder Geweben sekundär kritisch angereichert oder durch verbreitungsfördernde Mechanismen rasch verdünnt und verteilt werden und so fort. Zusammen mit der bekannten Tatsache, daß unsere unter konventionellen Bedingungen durchgeführten Laboratoriumsuntersuchungen vielfach nur in recht beschränktem Ausmaß Schlußfolgerungen über die tatsächlich zu erwartenden Wirkungen in situ erlauben, mahnen diese Oberlegungen zur Vorsicht bei der Anwendung einer jeden Globalklassifikation. 
Tomczak: Ich bin Thnen dankbar für diese Bemerkung. Tatsächlich muß man für die Praxis so verfahren, daß die Anwendung unseres Schemas nicht automatisch erfolgt, sondern daß wissenschaftliche Versuche und Überlegungen dabei ständig neu angestellt werden. Dies wird sicher auch aus dem Vortrag von Herrn WEICHART auf diesem Symposion über die chemischen Veränderungen bei Einleitungen hervorgehen. 